\title{
DESIGN, SYNTHESIS AND TARGETED DELIVERY OF FLUORESCENT 1,2,4- TRIAZOLE-PEPTIDE CONJUGATES TO PEDIATRIC BRAIN TUMOR CELLS
}

Muhammad Ajmal a, Uzma Yunus ${ }^{\mathrm{a}, *}$, Regina M. Graham ${ }^{\mathrm{b}}$, Roger M. Leblanc ${ }^{\mathrm{c}}$

a) Department of Chemistry, Allama Iqbal Open University, Islamabad, 44000, Pakistan

b) Department of Neurological Surgery, Miller School of Medicine, University of Miami, Miami,

Florida, 33136, United States

c) Department of Chemistry, University of Miami, 1301 Memorial Drive, Coral Gables, Florida, 33146, United States

\section{*Corresponding author:}

Department of Chemistry, Allama Iqbal Open University, Islamabad-44000, Pakistan

Tel. +92519057755, Fax: +92519250087

E-mail addresses: uzma_yunus@yahoo.com (Uzma Yunus)

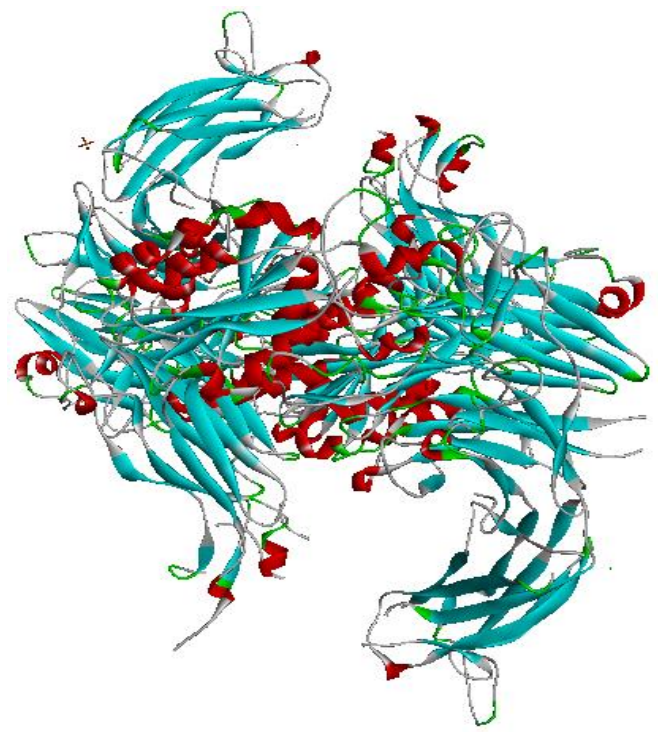

Figure S1. Optimized crystal structure of $\alpha v \beta 6$ integrin (PDB 4UM9) used for docking studies 

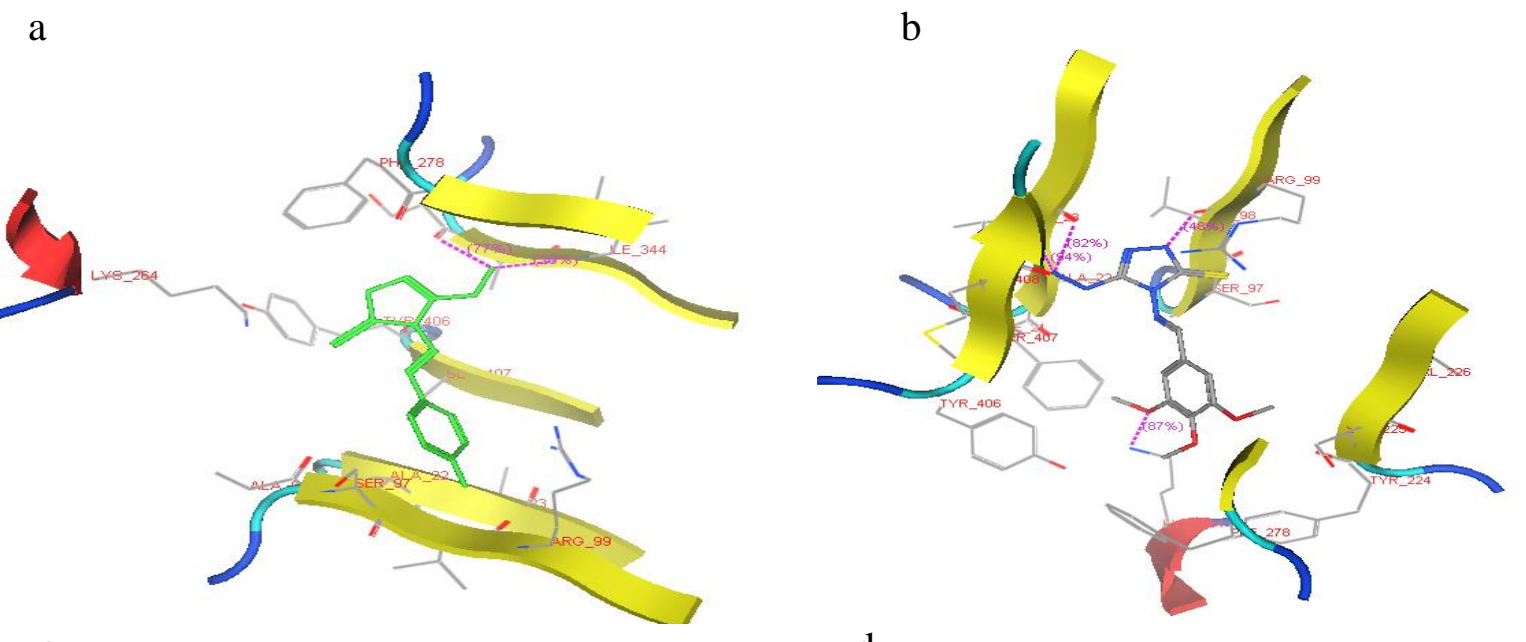

$\mathrm{c}$

d
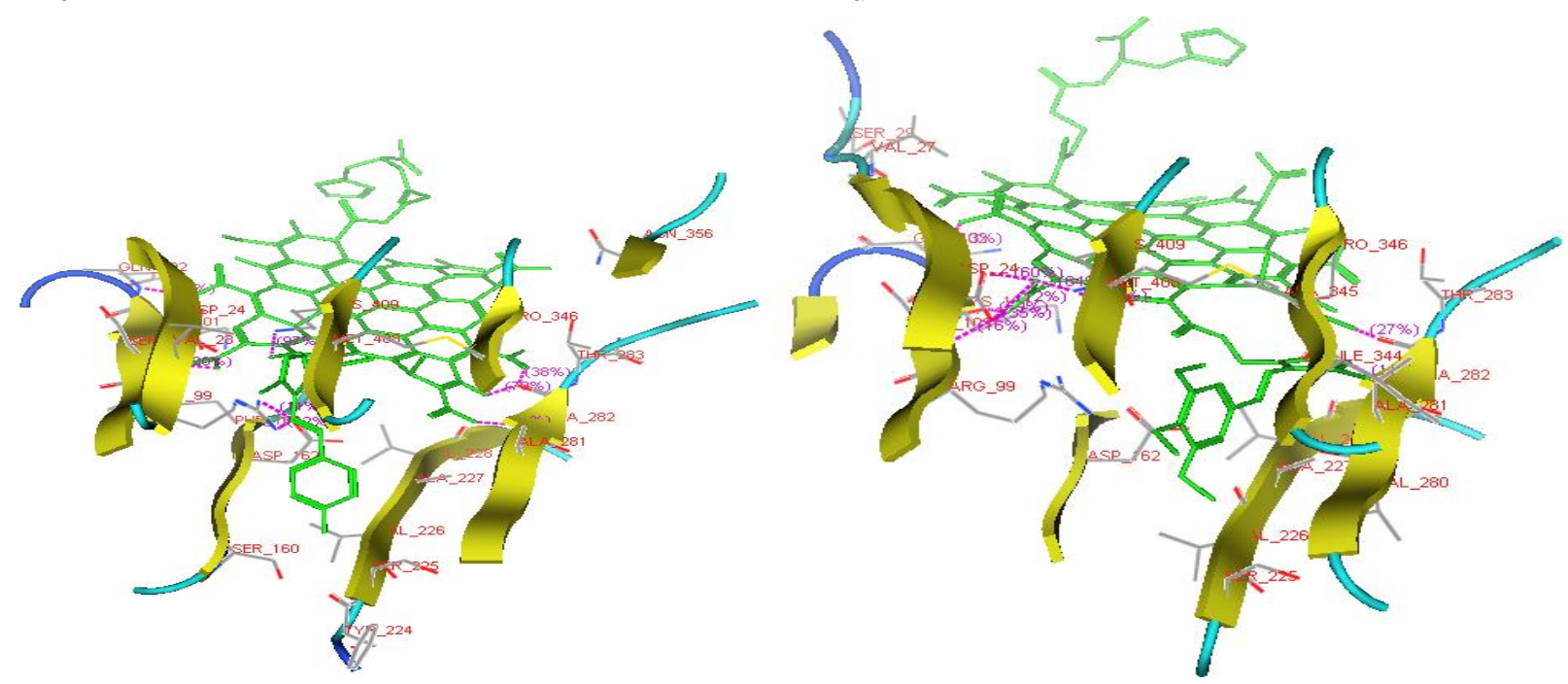

Figure S2. Docking presentation of (a, c) 4FBAHMT derivative and LC-CNP-4FBAHMT conjugate; (b, d) TMOBAHMT derivative and LC-CNP-TMOBAHMT conjugate, respectively in the active binding site of $\alpha \mathrm{v} \beta 6$ integrin

Supplementary Table S1. Physico-Chemical Properties of CNPs and LC-CNP4FBAHMT Conjugate

\begin{tabular}{|l|c|c|}
\hline Properties & CNPs (3) & LC-CNP-4FBAHMT Conjugate (4a) \\
\hline Physical appearance & solid powder & solid powder \\
\hline Color & black & brown \\
\hline Moisture (\%) & 53.31 & 41.60 \\
\hline Dry Content $(\boldsymbol{\%})$ & 46.69 & 59.40 \\
\hline Density $\left(\mathbf{g m} / \mathbf{c m}^{\mathbf{3}}\right)$ & 1.260 & 1.10 \\
\hline $\mathbf{p H}$ & $4.0-6.0$ & $6.0-7.0$ \\
\hline Solubility & water, ethanol & water, ethanol \\
\hline
\end{tabular}




\section{Electron Microscopy}

CNPs (3) and their conjugates (4a-c) were characterized by SEM, TEM and AFM microscopy for morphology, composition and size estimation. The SEM analysis of the samples for morphology study was performed by using JEOL JSM-6490 scanning electron microscope. SEM histograms of CNPs showed that these are mono-dispersed, nano-sized and uniform in size distribution (Figure S3a). The LC-CNP-4FBAHMT conjugates are also spherical and uniform in size. The conjugates are larger in size than CNPs (Figure S3b).
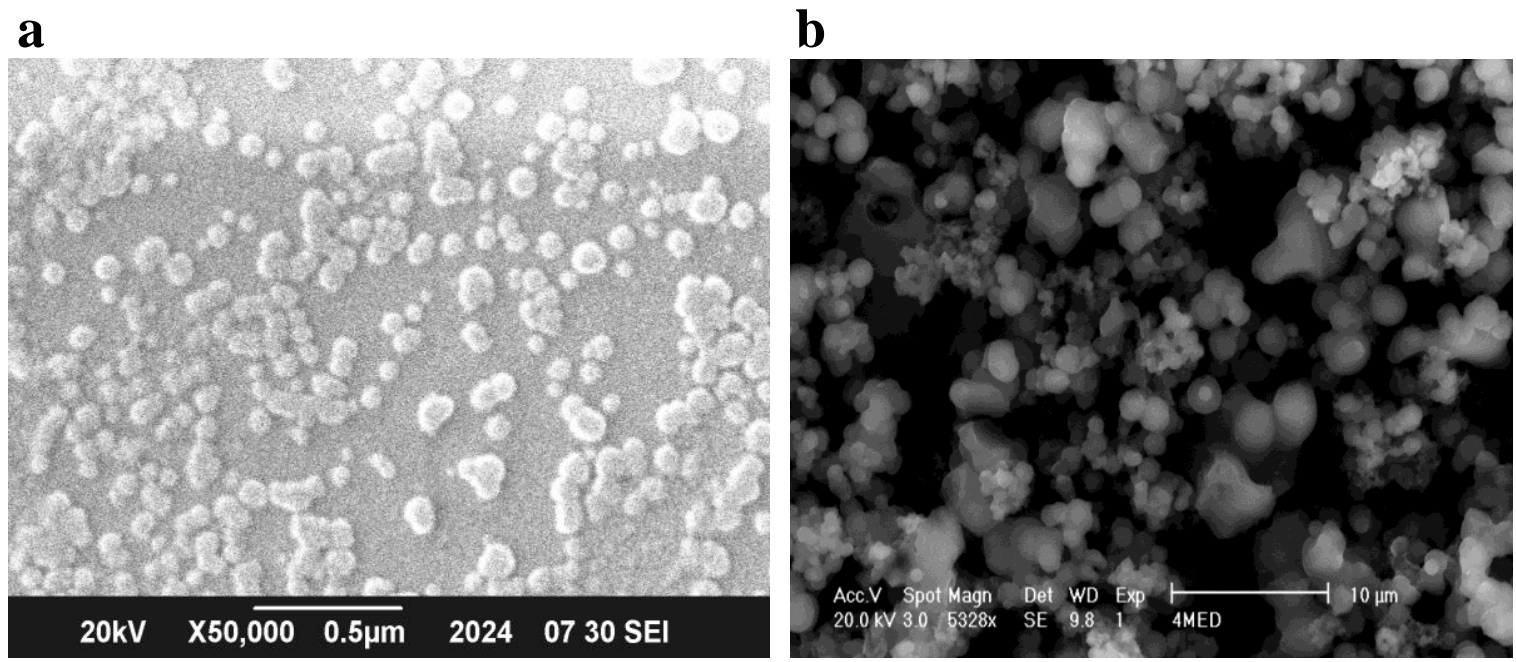

Figure S3. SEM images of (a) CNPs (b) LC-CNP-FBAHMT conjugate

\section{Energy Dispersive X-Ray Spectrometry (EDS) Analysis}

The elemental composition and the purity of the CNPs and LC-CNP-1,2,4-triazole conjugates were determined by using JEOL JSM-6490 scanning electron microscope. The microscope was operated at an acceleration voltage of $20 \mathrm{kV}$. The samples were cast as a thin film of the aqueous dispersion on an adhesive tape. The EDS histogram of CNPs indicated that carbon and oxygen are the main constituents of CNPs (Figure S4a and Supplementary Table S2). From this table, it can be observed that very high carbon (42\%) and oxygen (58\%) content are present in CNPs. The presence of sulphur in LC-CNP-4FBAHMT conjugates as shown by heavy metal EDS histogram confirmed the conjugation process of 4FBAHMT 1,2,4-triazole derivative with CNPs (Figure S4b). 


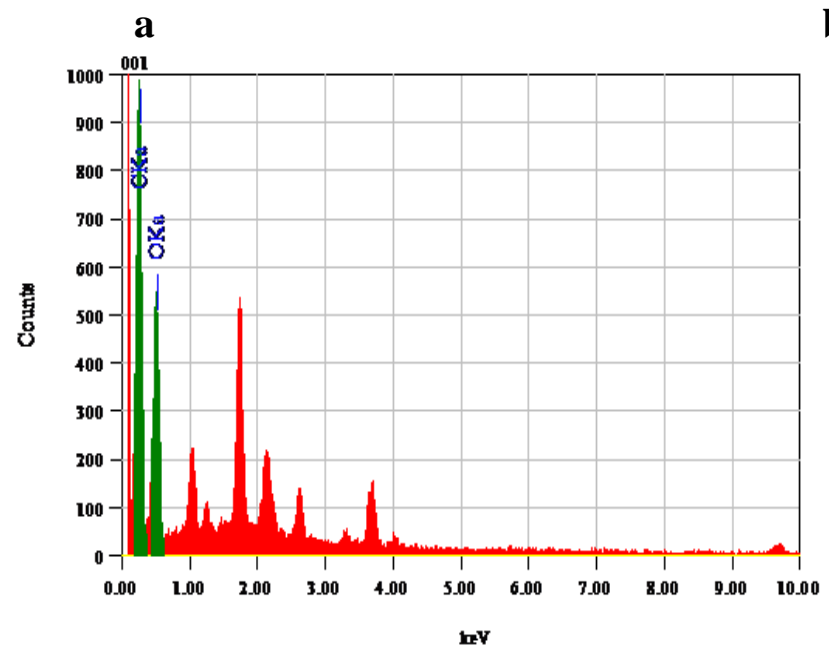

b

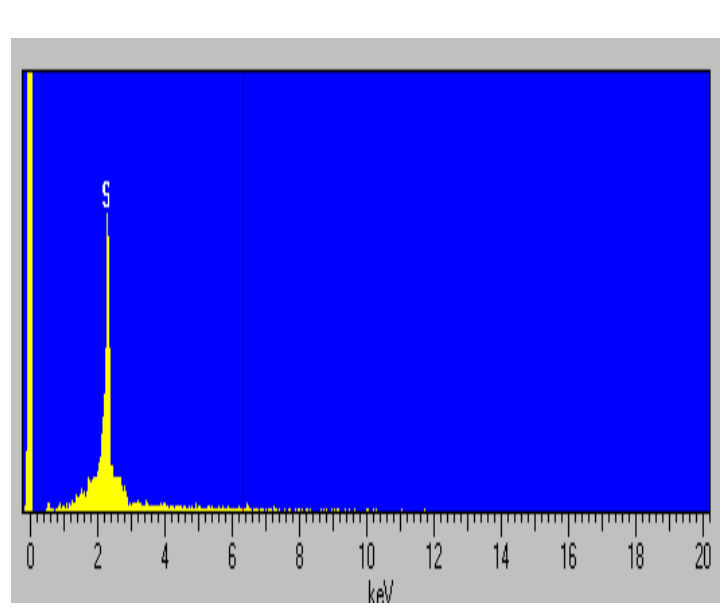

Figure S4. EDS histographs of (a) CNPs (b) LC-CNP-4FBAHMT conjugate

Supplementary Table S2. Elemental Composition of CNPs Studied by EDS Analysis

\begin{tabular}{|l|l|l|l|l|l|}
\hline Element & $(\mathbf{k e V})$ & Mass\% & Error\% & Atom\% & K \\
\hline & 0.277 & 41.840 & 0.560 & 48.930 & 36.924 \\
\hline & 0.525 & 58.160 & 2.650 & 51.070 & 63.076 \\
\hline & & $\mathbf{1 0 0 . 0 0 0}$ & & $\mathbf{1 0 0 . 0 0 0}$ & \\
\hline
\end{tabular}

\section{Characterization of LC-CNP-TMOBAHMT Conjugate (4b)}

The UV/Vis spectra of TMOBAHMT derivative showed two strong absorption peaks around 262 and $330 \mathrm{~nm}$ due to $\Pi-\Pi^{*}$ and $\mathrm{n}-\Pi^{*}$ transitions, respectively (Figure S5a). The LC-CNPTMOBAHMT conjugate showed red shift due to the extended conjugation resulting from amide bond formation between carboxylic groups of CNPs and amino groups of TMOBAHMT derivative and LC. In this conjugate, two peaks are observed at 272 and $345 \mathrm{~nm}$ due to this red shift. The strong absorption peaks of CNPs at 260 and $325 \mathrm{~nm}$ resulting from to $\Pi-\Pi^{*}$ and $\mathrm{n}-\Pi^{*}$ transitions are shifted to longer wavelengths in the conjugate. The LC-CNP-TMOBAHMT conjugate maintained absorbance properties after functionalization and conjugation process. 
Photoluminescence spectra of LC-CNP-TMOBAHMT conjugate showed the excitation dependent PL properties from 300 to $650 \mathrm{~nm}$ (Figure S5b). The functional group modifications and attachment of LC and TMOBAHMT derivative do not change the PL properties of CNPs. LC-CNP-TMOBAHMT conjugate showed strong PL from 300 to $650 \mathrm{~nm}$ with maximum emission at $500 \mathrm{~nm}$.

To further explore the fluorescence properties of LC-CNP-TMOBAHMT conjugate, PL spectra were recorded at different excitation wavelengths. Photoluminescence emission spectra were obtained with progressively longer wavelengths from 300 to $650 \mathrm{~nm}$.

The PL spectra showed in Figure S5b were recorded at individual excitation wavelengths of 250, 300, 350, 400, 450, 500, 550 and $600 \mathrm{~nm}$, respectively. The emission spectra were observed at wavelengths of 300, 350, 400, 450, 500, 550, 600 and $650 \mathrm{~nm}$, respectively.
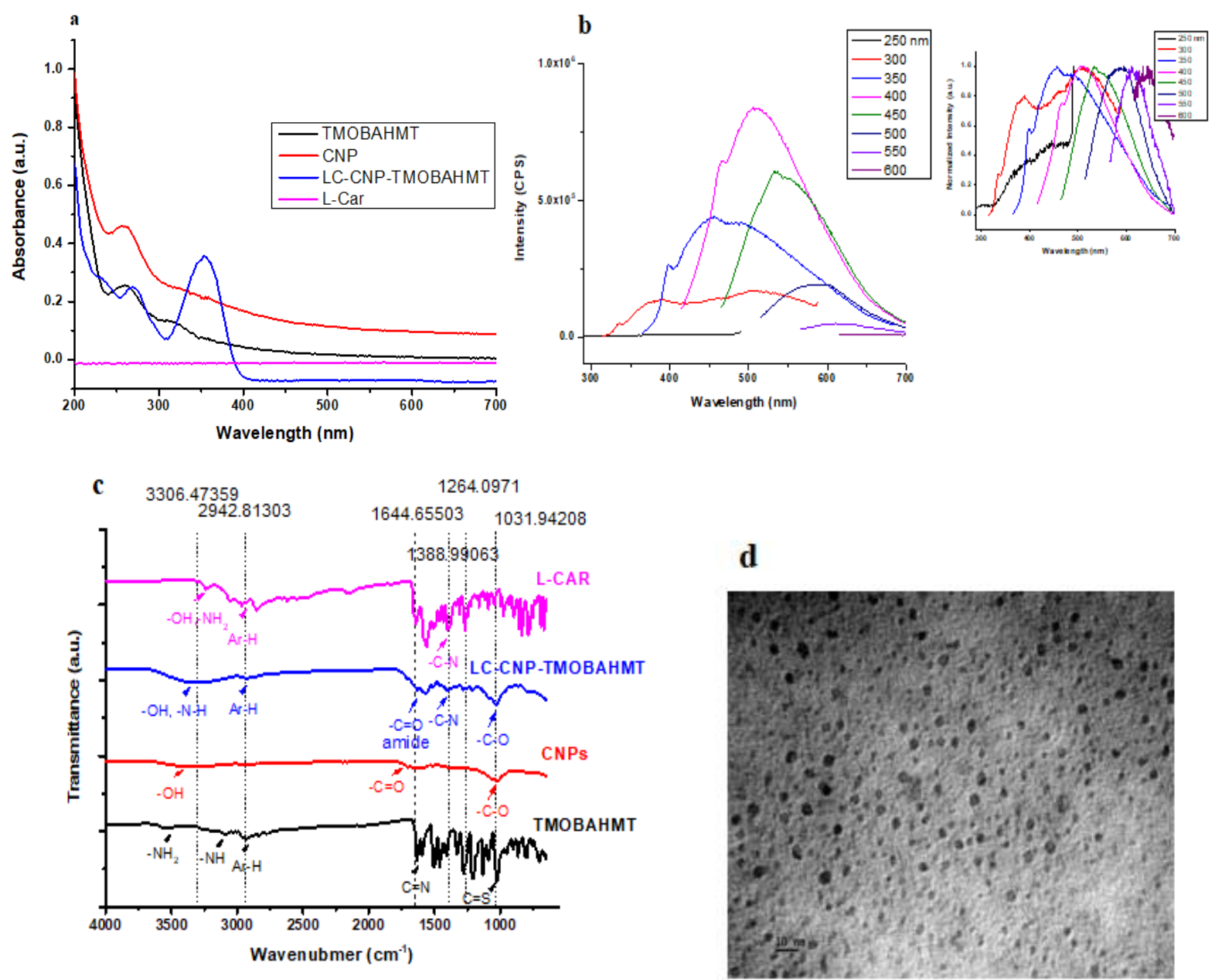

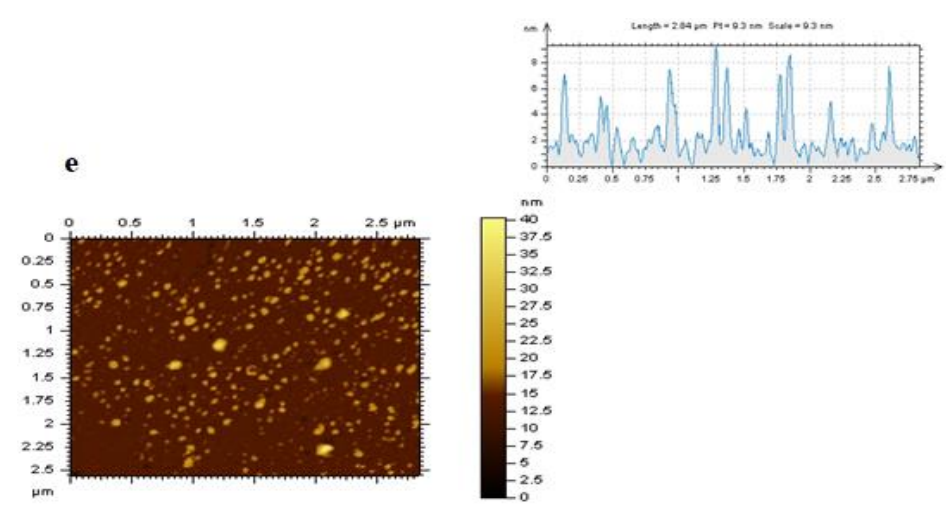

Figure S5. Spectroscopic and microscopic characterization of LC-CNP-TMOBAHMT conjugate: (a) UV-Vis spectra (b) PL spectra, inset is the normalized spectra (c) FTIR spectra (d) TEM image (e) AFM image, inset is the height profile of AFM image

The comparison of FTIR spectra of CNPs, LC, TMOBAHMT derivative and LC-CNPTMOBAHMT conjugate is given in Figure S5c. The bands at 1700 and $1620 \mathrm{~cm}^{-1}$, attributed to $\mathrm{C}=\mathrm{O}$ and $\mathrm{C}=\mathrm{C}$ stretching vibrational modes, respectively. Very small bands around 2150 and $3000 \mathrm{~cm}^{-1}$ are caused by $\mathrm{C}=\mathrm{C}$ and $\mathrm{C}-\mathrm{H}$ stretching. The bands in the range $1300-1000 \mathrm{~cm}^{-1}$, which include the $\mathrm{C}-\mathrm{OH}$ stretching and $\mathrm{O}-\mathrm{H}$ bending vibrations, and very broad and high intensity peak around $3400 \mathrm{~cm}^{-1}$ is attributed for $\mathrm{O}-\mathrm{H}$ stretching, imply the existence of large numbers of residual hydroxy groups. Low intensity vibrations around $570 \mathrm{~cm}^{-1}$ are characteristic peaks of CNPs.

The vibrational stretching bands between $3480-3020 \mathrm{~cm}^{-1}$ are characteristic for $\mathrm{NH}_{2}$ and $\mathrm{NH}$ groups of LC and TMOBAHMT derivative. Similarly, vibration bands around 1650 (C=O str.), 1600 (N-H bend), 1400 (C-N stretch) and $1200 \mathrm{~cm}^{-1}$ (C-O stretch) suggest the presence of LC. The presence of two stretching bands around $1620(\mathrm{~N}=\mathrm{CH})$ triazole ring and $1580 \mathrm{~cm}^{-1}$ $(\mathrm{N}=\mathrm{CH})$ imine are characteristic peaks of TMOBAHMT derivative.

The FTIR spectrum of LC-CNP-TMOBAHMT conjugate is also represented in Figure S5c. This spectrum shares some bands with the spectrum of CNPs on one hand and with spectra of $\mathrm{LC}$ and TMOBAHMT derivative on the other hand. The widening of $\mathrm{O}-\mathrm{H}$ stretching band between $3255-3245 \mathrm{~cm}^{-1}$ with moderate intensity and shifting of $\mathrm{C}=\mathrm{O}$ stretching band to lower 
frequency (around $1680 \mathrm{~cm}^{-1}$ ) showed the formation of amide bond in LC-CNP-TMOBAHMT conjugate. This band is caused by the presence of OH groups of CNPs and LC. The presence of $\mathrm{N}-\mathrm{H}$ bands plus unusually low value for the $\mathrm{C}=\mathrm{O}$ suggest the formation of an amide bond in $\mathrm{LC}$ CNP-TMOBAHMT conjugate.

The TEM and AFM microscopy results showed that the average size of LC-CNP-TMOBAHMT conjugate is 8-9 nm. TEM microscopy results (Figure S5d) were supported by AFM results and the extracted height profile (Figure S5e).

\section{Characterization of LC-CNP-4BO2MOBAHMT Conjugate (4c)}

The UV/Vis spectra of 4BO2MOBAHMT derivative (2c) showed two strong absorption peaks around 260 and $325 \mathrm{~nm}$ due to $\Pi-\Pi^{*}$ and $\mathrm{n}-\Pi^{*}$ transitions, respectively (Figure S6a). The LCCNP-4BO2MOBAHMT conjugate (4c) showed red shift due to the extended conjugation resulting from amide bond formation between carboxylic groups of CNPs and amino groups of 4BO2MOBAHMT derivative and LC. In this conjugate, two peaks are observed at 275 and 344 $\mathrm{nm}$ due to this red shift. The absorption peaks of CNPs at 260 and $325 \mathrm{~nm}$ resulting from to П-П* and $\mathrm{n}-\Pi^{*}$ transitions are shifted to longer wavelengths in the LC-CNP-4BO2MOBAHMT conjugate. The LC-CNP-4BO2MOBAHMT conjugate maintained absorbance properties after functionalization and conjugation process.

LC-CNP-4BO2MOBAHMT conjugate showed excitation dependent photoluminescence properties ranging from 300 to $650 \mathrm{~nm}$ with maximum emission at $500 \mathrm{~nm}$ (Figure S6b). The functional group modifications and attachment of LC and 4BO2MOBAHMT derivative with CNPs do not change the PL properties of CNPs.

To further explore the fluorescence properties of LC-CNP-4BO2MOBAHMT conjugate, PL spectra were recorded at different excitation wavelengths. Photoluminescence emission spectra were obtained with progressively longer wavelengths from 300 to $650 \mathrm{~nm}$. The PL spectra showed in Figure S6b were recorded at individual excitation wavelengths of 250, 300, 350, 400, $450,500,550$, and $600 \mathrm{~nm}$, respectively. The emission spectra were observed at wavelengths of $300,350,400,450,500,550,600$ and $650 \mathrm{~nm}$, respectively. 

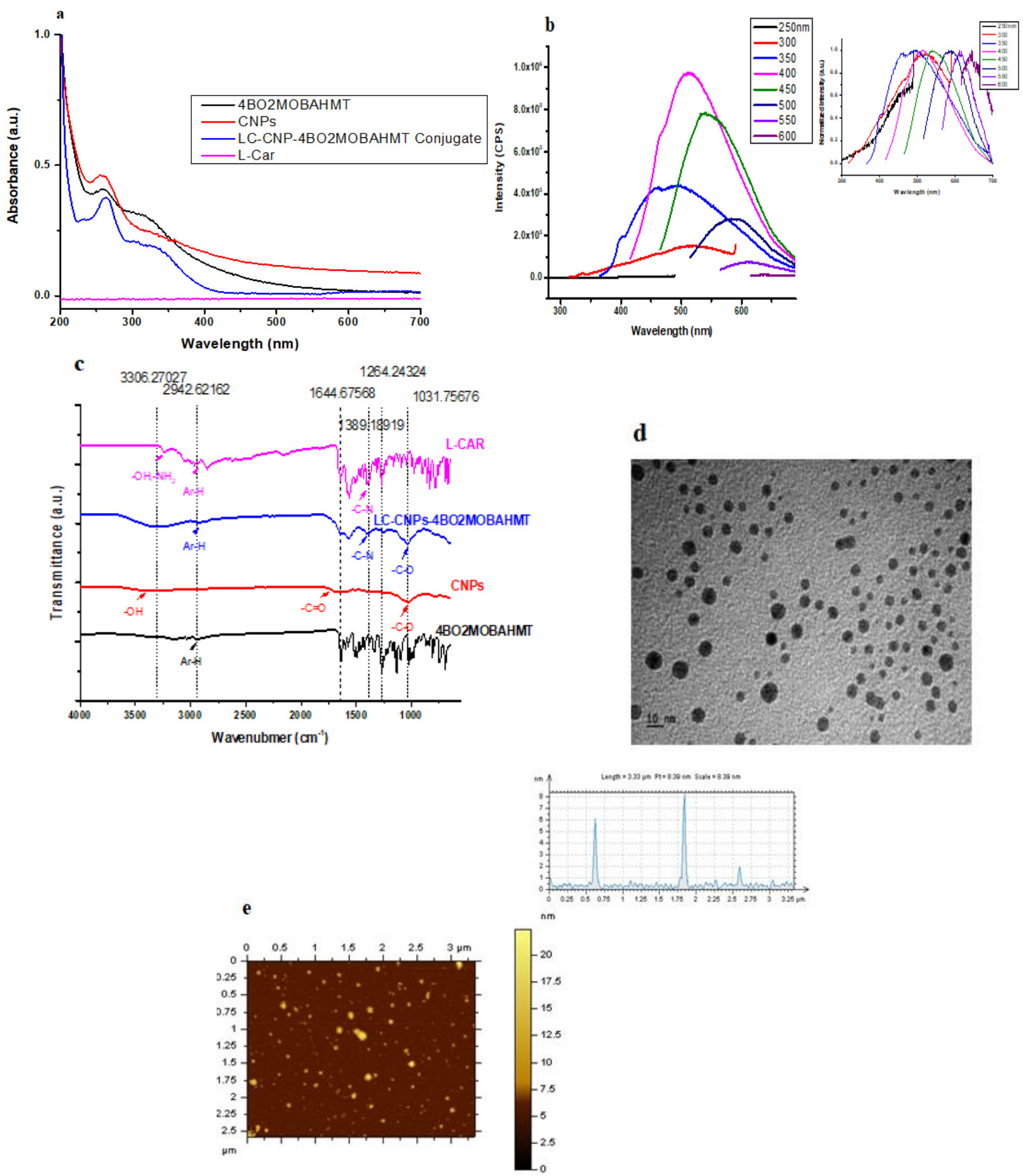

Figure S6. Spectroscopic and microscopic characterization of LC-CNP-4BO2MOBAHMT conjugate: (a) UV-Vis spectra (b) PL spectra, inset is the normalized spectra (c) FTIR spectra (d) TEM image (e) AFM image, inset is the height profile of AFM image 
The comparison of FTIR spectra of CNPs, LC, 4BO2MOBAHMT derivative and LC-CNP4BO2MOBAHMT conjugate is given in Figure S6c. The bands at 1700 and $1620 \mathrm{~cm}^{-1}$, attributed to $\mathrm{C}=\mathrm{O}$ and $\mathrm{C}=\mathrm{C}$ stretching vibrational modes, respectively, which are consistent with the UV-Vis absorption spectra. Very small bands around 2150 and $3000 \mathrm{~cm}^{-1}$ are caused by $\mathrm{C}=\mathrm{C}$ and $\mathrm{C}-\mathrm{H}$ stretching. The bands in the range $1300-1000 \mathrm{~cm}^{-1}$, which include the $\mathrm{C}-\mathrm{OH}$ stretching and $\mathrm{O}-\mathrm{H}$ bending vibrations, and very broad and high intensity peak around $3400 \mathrm{~cm}^{-1}$ is attributed for $\mathrm{O}-\mathrm{H}$ stretching, imply the existence of large numbers of residual hydroxy groups. Low intensity vibrations around $570 \mathrm{~cm}^{-1}$ are characteristic peaks of CNPs.

The vibrational stretching bands between $3380-3050 \mathrm{~cm}^{-1}$ are characteristic for $\mathrm{NH}_{2}$ and $\mathrm{NH}$ groups of LC and 4BO2MOBAHMT derivative. Similarly, vibration bands around $1650(\mathrm{C}=\mathrm{O}$ str.), 1600 (N-H bend), 1400 (C-N stretch) and $1200 \mathrm{~cm}^{-1}$ (C-O stretch) suggest the presence of LC. The presence of two stretching bands around $1620(\mathrm{~N}=\mathrm{CH})$ triazole ring and $1580 \mathrm{~cm}^{-1}$ $(\mathrm{N}=\mathrm{CH})$ imine are characteristic peaks of 4BO2MOBAHMT derivative.

The FTIR spectrum of LC-CNP-4BO2MOBAHMT conjugate is represented in Figure S6c. The spectrum of this conjugate shares some bands with the FTIR spectra of CNPs on one hand and LC and 4BO2MOBAHMT derivative on the other hand. The widening of $\mathrm{O}-\mathrm{H}$ stretching band between $3265-3250 \mathrm{~cm}^{-1}$ with moderate intensity and shifting of $\mathrm{C}=\mathrm{O}$ stretching band to lower frequency (around $1680 \mathrm{~cm}^{-1}$ ) showed the formation of amide bond in LC-CNP4BO2MOBAHMT conjugate. This band is due to the presence of OH groups of CNPs and LC. The presence of $\mathrm{N}-\mathrm{H}$ bands plus unusually low value for the $\mathrm{C}=\mathrm{O}$ suggest the formation of an amide bond in LC-CNP-4BO2MOBAHMT conjugates.

The TEM and AFM results showed that the average size of LC-CNP-4BO2MOBAHMT conjugate is $8 \mathrm{~nm}$. TEM microscopy results (Figure S6d) were supported by AFM results and the extracted height profile (Figure S6e). 


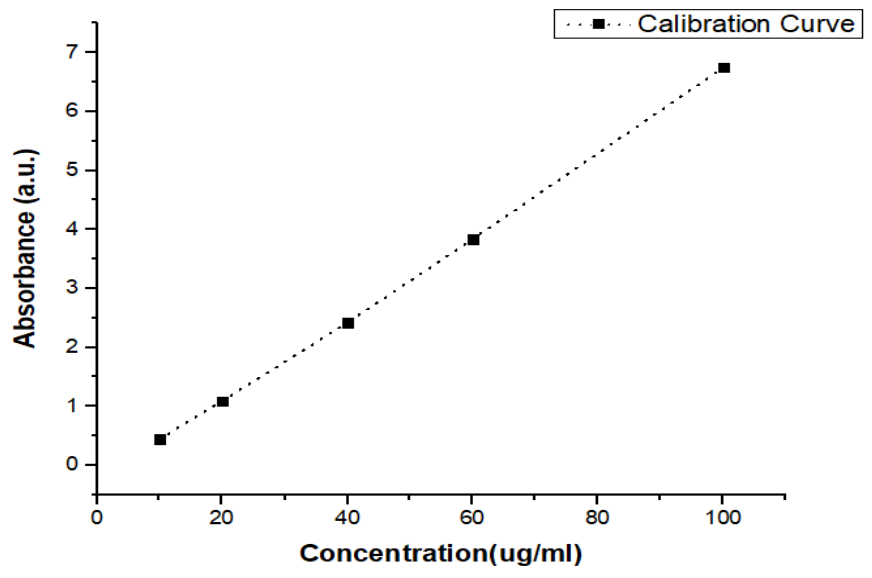

Figure S7. Calibration curve used for the determination of maximum drug loading 